Family Medicine and Community Health

\section{COVID-19 and beyond: how lessons and evidence from implementation research can benefit health systems' response and preparedness for COVID-19 and future epidemics}

To cite: Diop N, Kamal M, Renaud M, et al. COVID-19 and beyond: how lessons and evidence from implementation research can benefit health systems' response and preparedness for COVID-19 and future epidemics. Fam Med Com Health 2021;9:e001150. doi:10.1136/fmch-2021-001150
Check for updates

(C) Author(s) (or their employer(s)) 2021. Re-use permitted under CC BY-NC. No commercial re-use. See rights and permissions. Published by BMJ.

Global Health Division, International Development Research Centre, Ottawa, Ontario, Canada

Correspondence to Dr Nafissatou Diop; ndiop@idrc.ca

\section{ABSTRACT}

Early in the COVID-19 pandemic - and based on limited data on the novel coronavirus - it was projected that African countries will be ravaged and the health systems overwhelmed. Fortunately, Africa has so far defied these dire predictions. Many factors account for the less dramatic outcome, in particular the local know-how gained through dealing with previous epidemics, such as Ebola, and the early and coordinated political and public health response, applying a combination of containment and mitigation measures. However, these same measures, exacerbated by existing inequalities, have had negative impacts on vulnerable populations, notably women and children. Furthermore, the observed deterioration of access to and provision of essential health services will likely continue and worsen in countries experiencing future waves of COVID-19 and lacking access to vaccines. The impact of the pandemic on health systems may be one of Africa's main COVID-19 challenges and women and children its greatest victims. In this article, we argue that just as learning from previous epidemics and coordinated preparation informed Africa's response to COVID-19, knowledge, innovations and resources from recent implementation research can be leveraged to mitigate the pandemic's effects and inform recovery efforts. As an example, we present the proven model and multifaceted approach of the Innovating for Maternal and Child Health in Africa Initiative and describe how such a model could be readily applied to building the robust and equitable systems needed to tackle future stresses and shocks, such as epidemics, on health systems while maintaining essential routine services.

\section{INTRODUCTION}

Early in the pandemic's first wave-and based on limited data on the novel coronavirusthe United Nations Economic Commission for Africa warned that COVID-19 could kill up to 300000 during the first year in African countries and that the number of cases would overwhelm available medical capacity. ${ }^{1}$ Fortunately, Africa has defied these dire predictions, and as of 16 April 2021, a year into the pandemic, the official cumulative death count for Africa was $115840 .^{2}$

Many factors can account for the discrepancy, in particular the know-how gained through dealing with previous epidemics and disease outbreaks, such as Ebola. ${ }^{34}$ The early and coordinated political and public health response, applying a combination of containment and mitigation measures, effectively helped Africa's swift response to COVID-19 and contributed to limiting transmission. ${ }^{5}$

However, these same measures, exacerbated by existing inequalities, have had negative impacts on vulnerable populations, notably women and children who suffer most from shocks and stresses to health systems. ${ }^{6}$ For example, a review of health, economic and social systems that result in genderbased differences in various areas such as the healthcare workforce, reproductive health, gender-based violence and mental health during the COVID-19 pandemic found that female caregivers have an increased exposure risk of contracting SARS-CoV-2, the virus causing COVID-19. COVID-19 has also restricted access to family planning and intimate partner violence resources. Furthermore, the authors report that 'multifactorial stress is uniquely exacerbated among women during COVID-19'. ${ }^{\prime}$

In an effort to curb the pandemic as quickly as possible, health systems' resources pivoted to COVID-19, undermining the delivery of other essential health services. ${ }^{8}$ The observed deterioration of access to and provision of essential health services will likely continue and worsen in countries experiencing second, third and future waves of COVID-19 and lacking access to vaccines. The impact of the pandemic on health systems may be one of Africa's main COVID-19 challenges 
and women and children its greatest victims. Preparing for COVID-19 resurgence, and other epidemics, in Africa requires empowering communities to be first responders, assessing risk at appropriate administrative levels and planning for worst-case scenarios by understanding and supporting health system responses. ${ }^{9}{ }^{10}$ Leveraging past research provides a shortcut to the requirements for mounting an appropriate evidence-informed response.

\section{LEVERAGING PAST RESEARCH TO INFORM CONTINUITY OF ESSENTIAL SERVICES AS PART OF THE RESPONSE TO AN EPIDEMIC}

Just as learning from previous epidemics and coordinated preparation informed Africa's response to COVID-19, so can knowledge, innovations and resources from health implementation research be leveraged to mitigate the pandemic's effects and inform recovery efforts. Implementation research is defined as 'the scientific study of the processes used in the implementation of initiatives as well as the contextual factors that affect these processes. It can address or explore any aspect of implementation, including the factors affecting implementation..., the processes of implementation themselves... and the outcomes,or end-products of the implementation under study.'. ${ }^{11}$ One specific example is the Innovating for Maternal and Child Health in Africa (IMCHA) Initiative. Cofunded by Global Affairs Canada, the Canadian Institutes of Health Research and Canada's International Development Research Centre, IMCHA is an 8-year (2014-2021), \$C36million initiative to improve access to quality services for reproductive, maternal, newborn and child health services in Africa, using primary healthcare as the entry point. IMCHA focused on four priority themes: high-impact community-based interventions, quality facility-based interventions, policy environment to improve care services and outcomes, and human resources. The initiative funded 19 research teams working on 28 projects in 11 countries in sub-Saharan Africa (Burkina Faso, Ethiopia, Kenya, Malawi, Mali, Mozambique, Nigeria, Senegal, South Sudan, Tanzania and Uganda). IMCHA also supported two regional health policy and research organisations (HPROs) to work with the research teams in capacity strengthening and knowledge translation to raise the profile of the research and facilitate uptake of the findings in national and regional policies and practices by higher-level decision-makers than research teams can usually reach on their own. ${ }^{12}$

Each IMCHA research team is led by an African principal investigator, working closely with two coprincipal investigators: one researcher from Canada and an African decision-maker. This partnership, a collaboration between researchers and decision-makers, allows targeting the most pressing needs in each country and context. Furthermore, the collaboration between research teams and HPROs contributes to concomitantly expand the reach of research findings to very high-level decision-makers for evidence-informed policy and practice. The result demonstrated better health outcomes for the population in research settings and beyond, particularly mothers and children. One example is more pregnant women in Tanzania, Mali or Mozambique are now able to get health services in a timely manner because the implementation of national policies was revised. Another example is women in remote areas without easy access to a health facility in Nigeria, Senegal, Tanzania or Uganda are now informed of or monitored for danger signs during pregnancy by better trained, equipped and motivated community health workers right in their communities for better outcomes for the mother and the newborn.

IMCHA offers a proven model of how research can be mobilised to strengthen health systems. As the examples demonstrate, the model supports finding context-specific solutions to health problems, based on locally generated evidence and capitalising on lessons learnt. It can be easily adapted to inform how to respond to and prepare for epidemics and disease outbreaks while maintaining provision of essential health services during and after such disruptions wherever they happen.

We summarise here lessons in four areas that are at the heart of a pandemic response: engaging decision-makers, involving communities, strengthening health systems and building on successful interventions and collaborations.

\section{Engaging decision-makers}

Integrating a decision-maker in research projects early on and at the right authority level ensures local relevance and ownership over the process and the solutions, strong policy buy-in and continued engagement for problem solving and adoption of solutions. This has fast-tracked the uptake of successful solutions into policy and practice. Decision-makers identify priority questions and policy gaps and facilitate adoption of research findings.

For example, in Oyo State, Nigeria, the Ministry of Health incorporated mental health screening and treatment tools, developed and implemented between 2017 and 2020 by an IMCHA research team, into the routine assessment of pregnant women receiving antenatal care. ${ }^{13}$ This improved mental health outcomes for the normally underserved perinatal women living with depression. For example, the research team reported that the intervention, delivered from the second trimester through to the postnatal period, produced significantly better remission from perinatal depression compared with treatment as usual (control arm), with $37.0 \%$ remission in the control arm and $55.7 \%$ remission in the intervention arm at the 6 -month postnatal assessment $(\mathrm{p}<0.005)$. Furthermore, for the first time, mental health desks have been established at the state and federal levels, providing substantive evidence that innovations and system enhancements can be integrated into pre-existing government structures, even in low-resource settings. This model could help meet the additional demand for mental health services seen as a result of epidemics, such as COVID-19, close to where people are. 
At national and regional levels, the HPROs supported the research teams to take their findings beyond their usual limited reach, paving the way for largerscale geographical and policy impact. For example, in Tanzania, the East Africa HPRO convened several forums providing the six IMCHA research teams in the country prime access to high-level decision-makers to present evidence of inappropriate policies and practices within the health system. Among other successes, the teams' recommendations led to renewed government commitment to facilitate women's access to antenatal services, such as by ensuring that health providers do not make the presence of the male partner of a pregnant woman a condition for receiving respectful and timely antenatal services. ${ }^{14}$ On the other side of the continent, at the 18 th Ordinary Session of the Assembly of Health Ministers of the 15 member states of the Economic Community of West African States (ECOWAS) in June 2017, the West Africa HPRO introduced the 'Resolution on the use of evidence in developing health care policies, plans, standards and protocols in the ECOWAS region'. The Ministers adopted the resolution. The ministries are now committed to implementing it and are taking steps to do so. ${ }^{1516}$

IMCHA's approach to bridging the research-policy gap and building buy-in from the start is a good model that shows how the disconnect between decision-makers, at different levels of government, and scientific experts, witnessed in COVID-19 responses in many countries, could be overcome.

\section{Involving communities}

Communities are essential stakeholders regarding their own health. Appropriately engaging the communityworking with and learning from adolescents, women, men and community leaders-in defining and implementing relevant solutions is key to changing harmful social norms and to reaching successful outcomes. Community engagement also fosters mutual understanding and trust between populations and service providers, and it strengthens the accountability of health facilities. In addition, community consultations lead to a consensus on what research is needed and what the priorities are.

For example, in South Sudan, a research project used participatory, community-based interventions to work with existing women's groups. Trained and trusted facilitators led the groups of women in identifying priorities and developing strategies they could carry out with the support of local community members. For example, some groups chose to fight malnutrition by growing nutritious foods, while others repaired roads to improve access to healthcare services and schools. As a result, women gained confidence and leadership abilities, adopted healthier behaviours and had greater confidence in speaking publicly and approaching health professionals. ${ }^{17}$

IMCHA's strong inclusive approaches to communitycentred problem analysis and development of solutions fostered the commitment of communities and enhanced engagement and ownership of responses. Such approaches could help overcome resistance to prevention measures required to curb epidemics such as COVID-19 by, for example, helping mitigate harm from misinformation and facilitating vaccine acceptance.

\section{Strengthening health systems}

Human resources is a key building block of any health system. Ensuring healthcare workers' availability and capacity to provide quality services, including in times of disruption, is essential to better health outcomes. Several IMCHA projects identified gaps in healthcare workers' knowledge and practice and developed training tools and approaches to improve quality of services, which would be relevant for COVID-19 response. One project in Tanzania, for example, identified and addressed capacity gaps for healthcare workers in remote areas and developed a new e-learning curriculum, including training in comprehensive emergency obstetric and neonatal care, anaesthesia and newborn resuscitation. The Tanzania Ministry of Health embarked on a large-scale roll out of this curriculum to bring comprehensive emergency obstetric care to 320 health facilities in the country. ${ }^{18}$ Training like this makes it feasible to allow task shifting, increasing the range of services offered in primary health centres and reducing the need for referrals to the usually more costly higher levels of care. This could be crucial to increasing health systems' flexibility and targeting services to those most in need during COVID-19 and other health crises.

Effective health systems also need adequate infrastructure. Some low-cost solutions can be effectively implemented. Many primary healthcare facilities in sub-Saharan Africa are underfunded and poorly equipped. In three district health centres in Malawi, for example, a research team found a shortage of equipment, supplies and setup for newborn care in the labour ward, the surgical theatre and the nursery. To address the shortcomings, for premature babies in particular, the research team introduced an integrated healthcare package that includes simple initiatives, such as breastfeeding and skin-to-skin care for newborns, to improve their survival rate, as well as a low-cost, mechanical continuous positive airway pressure device to help newborns in respiratory distress. ${ }^{19}$

In addition, interventions to improve the readiness of health facilities to deal with epidemics and ensure the protection of health workers and the public are critical. For example, IMCHA projects introduced ways to improve the sanitary environment during labour. In one project in Tanzania, for instance, the interventions led to increases in key personal protective equipment availability from $14 \%$ at the start to $87 \%$ by the end of the intervention. Handwashing in the delivery area increased from $49 \%$ to $84 \% .^{20}$

The experience and expertise gained in health systems strengthening through initiatives such as IMCHA could be readily applied to building the robust, equitable systems needed to limit transmission and deliver services safely during epidemics such as COVID-19. 


\section{Building on successful interventions and collaborations}

Learning from interventions that have successfully addressed health system challenges can contribute to strengthening their resilience, so they are prepared to tackle sudden stresses and shocks while maintaining essential routine services. This is critical for preserving the confidence of local populations in their health services that took years to build and avoiding a 'yo-yo' phenomenon dragging down improvements in health outcomes after any major disruption. The IMCHA Initiative and above examples provide insights on ways to tackle similar challenges in other regions within a country, more broadly in Africa, and beyond.

As demonstrated by the global effort in the search for a vaccine against SARS-CoV-2, international collaboration is key for mounting a successful response to global threats and diseases. The collaboration IMCHA featured between African and Canadian researchers proved successful in building avenues for scientific exchange and mutual learning. Furthermore, the multidonor partnership allowed each funder to go above and beyond what they would be able to achieve on their own. The COVID-19 funders community can join forces to support research and the generation of sound evidence, as well as knowledge sharing for building health systems that are more resilient to current and future shocks. Furthermore, individual funders' contributions will yield an overall return on investment greater than the sum of the funding.

\section{CONCLUSION}

Building health systems and communities that are resilient to major disruptions, such as the COVID-19 pandemic, requires more than responding by shifting investment into medical countermeasures. IMCHA's successes in strengthening health systems, community mobilisation, leadership and intersectoral collaboration to reach vulnerable groups demonstrate that implementation research has a key role to play in informing effective solutions to sustaining essential services, supplies and medicines; supporting communities with information and services; and strengthening leadership to better prepare for and respond to emergencies. Donors, researchers, decisionmakers and other stakeholders in global health should leverage the full potential of implementation research and design innovative models like IMCHA. The benefits of medium-term and long-term, sustainable actions on public health should not be neglected in favour of immediate and short-term measures.

Acknowledgements This opinion piece is written by staff of Canada's International Development Research Centre (IDRC), which along with Global Affairs Canada and the Canadian Institutes of Health Research fund the Innovating for Maternal and Child Health in Africa (IMCHA) Initiative. The authors would like to thank Michelle Hibler, Francine Sinzinkayo and Nola Haddadian for their valuable input on earlier versions of the article. We also thank the IMCHA grantees and collaborating stakeholders in Africa and Canada, as well as the governments and populations of involved countries without which the IMCHA Initiative would not have been able to contribute to improving the lives of women and children.

Contributors ND: conceptualisation/design, literature search and writing. MK: conceptualisation/design, literature search and writing/editing. MR: information collection, literature search and writing/editing. SN: literature search and writing/ editing.

Funding The authors have not declared a specific grant for this research from any funding agency in the public, commercial or not-for-profit sectors.

Disclaimer The views expressed in this article are those of the authors alone and do not represent the views of the Innovating for Maternal and Child Health in Africa Initiative, the International Development Research Centre, the Canadian Institutes of Health Research, nor Global Affairs Canada.

Competing interests None declared.

Patient consent for publication Not required.

Provenance and peer review Not commissioned; externally peer reviewed.

Open access This is an open access article distributed in accordance with the Creative Commons Attribution Non Commercial (CC BY-NC 4.0) license, which permits others to distribute, remix, adapt, build upon this work non-commercially, and license their derivative works on different terms, provided the original work is properly cited, appropriate credit is given, any changes made indicated, and the use is non-commercial. See: http://creativecommons.org/licenses/by-nc/4.0/.

ORCID iD

Nafissatou Diop http://orcid.org/0000-0003-0264-3790

\section{REFERENCES}

1 United Nations, Economic Commission for Africa (UNECA). COVID-19 in Africa: protecting lives and economies. Addis Ababa, Ethiopia: UNECA, 2020. https://repository.uneca.org/handle/10855/43756

2 Africa CDC. Coronavirus disease 2019 (COVID-19). Addis Ababa, Ethiopia: African Union, 2019. https://africacdc.org/covid-19/

3 Miller NP, Milsom P, Johnson G, et al. Community health workers during the Ebola outbreak in Guinea, Liberia, and Sierra Leone. J Glob Health 2018;8:020601.

4 Wagenaar BH, Augusto O, Beste J, et al. The 2014-2015 Ebola virus disease outbreak and primary healthcare delivery in Liberia: timeseries analyses for 2010-2016. PLoS Med 2018;15:e1002508.

5 WHO. Opening statement, COVID-19 press conference, 24 September 2020. remarks by WHO regional director for Africa, Dr Matshidiso Moeti. Available: https://www.afro.who.int/regionaldirector/speeches-messages/opening-statement-covid-19-pressconference-24-september-2020

6 Vellekoop M, Achola M, Hanne J. Falling through the cracks: COVID-19 and the rise of maternal deaths in Africa. Think Global Health, 2020. Available: https://www.thinkglobalhealth.org/article/ falling-through-cracks-covid-19-and-rise-maternal-deaths-africa

7 Connor J, Madhavan S, Mokashi M, et al. Health risks and outcomes that disproportionately affect women during the Covid-19 pandemic: a review. Soc Sci Med 2020;266:113364.

8 Lal A, Erondu NA, Heymann DL, et al. Fragmented health systems in COVID-19: rectifying the misalignment between global health security and universal health coverage. Lancet 2021;397:61-7.

9 Impouma B, Mboussou F, Kasolo F, et al. Preparing for a COVID-19 resurgence in the WHO African region. Lancet 2021;397:373.

10 Desborough J, Dykgraaf SH, Phillips C. Lessons for the global primary care response to COVID-19: a rapid review of evidence from past epidemics, family practice, 2021. Available: https://academic.oup.com/ fampra/advance-article/doi/10.1093/fampra/cmaa142/6136179

11 Peters DH, Tran NT, Adam T. Implementation research in health: a practical guide. Geneva: WHO, 2013. https://www.who.int/alliancehpsr/alliancehpsr_irpguide.pdf

12 IDRC. Innovating for maternal and child health in Africa. Available: https://www.idrc.ca/en/initiative/innovating-maternal-and-childhealth-africa

13 Stadnick NA, Sadler E, Sandall J, et al. Comparative case studies in integrated care implementation from across the globe: a quest for action. BMC Health Serv Res 2019;19:899.

14 hera. Innovating for maternal and child health in Africa (IMCHA) summative evaluation, main report (Vol 1), 25 September 2020, p.18. hera tender template 05302018 (dspacedirect.org).

15 Diop N, Rashid A. Champions of evidence play a pivotal role in bridging research-to-policy gap. IDRC, 2019. Available: https://www. 
idrc.ca/en/perspectives/champions-evidence-play-pivotal-rolebridging-research-policy-gap

16 Sombie I, Johnson E, Moukaïla A. Promoting knowledge transfer and culture of evidence use for health systems strengthening in West Africa, 2018. Available: https://www.researchgate.net/publication/ 329118911_Promoting_knowledge_transfer_and_culture_of evidence_use_for_health_systems_strengthening_in_West_Africa

17 IDRC. Strengthening maternal and child health in conflict-affected South Sudan, 2020. Available: https://www.idrc.ca/en/research-inaction/strengthening-maternal-and-child-health-conflict-affectedsouth-sudan
18 Dol J, Campbell-Yeo M, Bulemela J, et al. Knowledge acquisition after helping babies survive training in rural Tanzania. Int Health 2019;11:136-42.

19 Kawaza K, Kinshella M-LW, Hiwa T, et al. Assessing quality of newborn care at district facilities in Malawi. BMC Health Serv Res 2020;20:227.

20 Matovelo D, Brenner J, Shabani G. Regional maternal newborn and child health strengthening in Tanzania. Endline study report, 2020. Available: https://www.mnmtanzania.com/wp-content/uploads/2020/ 07/MnMEndofProjectEvaluation_29-Jun-2020_FINAL.pdf [Accessed 12 Aug 2021]. 